\title{
Caracterização do envelhecimento populacional no município do Rio de Janeiro: contribuições para políticas públicas sustentáveis
}

\author{
Demographic aging characterization in the city of Rio de \\ Janeiro: contributions to sustainable public policies
}

\author{
Davi da Silveira Barroso Alves ${ }^{1}$, Maria Tereza Serrano Barbosa², \\ Ernesto Raúl Caffarena ${ }^{3}$, Alexandre Sousa da Silva ${ }^{2}$
}

\begin{abstract}
Resumo
Objetivo: Descrever o estágio do envelhecimento populacional no município do Rio de Janeiro. Métodos: Estudo ecológico tendo como unidades de observação os 160 bairros que compõem o município, utilizando indicadores sociais e demográficos construídos a partir de informações do Censo 2010. Realizou-se análise exploratória por meio de mapas temáticos e determinou-se a dependência espacial pelo Índice de Moran Global. Para agrupar bairros em estágios semelhantes do envelhecimento foi realizada uma análise de agrupamento a partir do método K-means. Resultados: Encontraram-se três grupos de bairros em estágios diferentes de envelhecimento populacional, identificando-se uma tendência espacial no sentido oeste-leste com os bairros da "Zona Sul" se encontrando no estágio mais avançado de envelhecimento. Conclusão: $O$ estudo identificou as diferenças no processo de envelhecimento populacional e na composição etária dos bairros, apontando para a necessidade de políticas de saúde pública específicas que contemplem as particularidades desse processo em cada localidade, visando garantir um envelhecimento sustentável. Palavras-chave: envelhecimento da população; políticas públicas de saúde; análise espacial.
\end{abstract}

\begin{abstract}
Objective: Describe the stage of population aging in the city of Rio de Janeiro. Methods: Ecological study with the 160 city neighborhoods as observational units, using social and demographic indicators built with information from the 2010 census. The exploratory analysis was undertaken with thematic maps, and the spatial dependence was measured with the Global Moran's Index. K-means clustering was used for grouping neighborhoods with similar aging stages. Results: Three neighborhood clusters in different stages of population aging were found and a spatial trend in the west-east direction was identified, with neighborhoods in the 'South Zone' in a more advanced stage of population aging. Conclusion: The study identified differences in the population aging process and in the age composition of neighborhoods, indicating the need for specific public health policies that allow for the particularities of this process in each location, aiming a sustainable population aging.
\end{abstract}

Keywords: population aging; public health policies; spacial analysis. 


\section{INTRODUÇÃO}

Nas últimas décadas, diversas regiões do mundo passaram por um processo de Transição Demográfica no qual populações, tipicamente jovens e adultas, tornaram-se gradualmente envelhecidas ${ }^{1,2}$. Essa transição e suas consequências para os sistemas de saúde, de seguridade e também para as cidades têm sido estudadas nos mais diversos países, que buscam antecipar os seus efeitos e planejar o futuro ${ }^{3}$.

Segundo Veras ${ }^{4}$, os desafios que o envelhecimento populacional impõe demandam uma mudança na lógica assistencial do sistema de saúde que, atualmente, prioriza o tratamento dos problemas de saúde existentes de maneira mais intensa do que ações de prevenção. Nessa mesma direção, os países em estágios avançados da transição demográfica têm se preocupado em identificar os principais desafios colocados para que, daqui a 20 anos, sejam superadas as dificuldades da sustentabilidade de um sistema de saúde onde o corpo profissional e os recursos financeiros poderão ser insuficientes se não houver prevenção de doenças crônicas nem medidas que afetem diretamente as condições de vida, emprego e outros determinantes sociais da saúde ${ }^{5}$.

No Brasil, esse processo vem ocorrendo em alta velocidade e nem as cidades, nem o Sistema de Saúde conseguiram ainda adaptar-se de forma a gerar qualidade de vida ou atender a população idosa, grupo populacional mais vulnerável e que mais utiliza o sistema de saúde ${ }^{6-8}$. Se, por um lado, as alterações sociais, econômicas e culturais, bem como os avanços médico-sanitários, determinaram a queda da mortalidade em geral e o aumento da expectativa de vida da população, por outro, uma proporção cada vez maior dessa população alcança idade mais avançada, sofrendo de doenças crônicas, que possuem período de latência e duração longos, podendo algumas delas deixar sequelas que impõem limitações às funções do indivíduo ${ }^{9,10}$, acarretando anos de utilização recorrente dos serviços de saúde.

Nesse contexto, ao considerar que uma das consequências desse processo de envelhecimento é o aumento na morbimortalidade por doenças crônicas e não transmissíveis, é natural que as políticas públicas adotem estratégias diferenciadas e ações regionais específicas, com base nos dados epidemiológicos e demográficos de cada unidade da federação, que permitam, por exemplo, verificar a associação entre dados de mortalidade e indicadores socioeconômicos ${ }^{11,12}$.

No mundo desenvolvido, Japão e Alemanha produziram, nos últimos anos, reformas no seu sistema de saúde com o objetivo de atender às mudanças ocorridas tanto devido ao declínio das doenças transmissíveis como ao envelhecimento da população $0^{13,14}$. O Brasil, como país em vias de desenvolvimento, tende a apresentar um processo de transição demográfica polarizado, marcado tanto pelas diferenças regionais quanto por sobreposição de causas de morbimortalidade. Elevadas taxas de mortalidade por doenças infecciosas acompanham o aumento das doenças crônicas ${ }^{15,16}$, o que torna emergencial discutir como se dará o fortalecimento e a sustentabilidade do Sistema Único de Saúde frente a esse processo.

Poucos estudos têm abordado as consequências no cenário urbano do envelhecimento populacional, contudo é de se esperar que a descrição das desigualdades que demarcam esse processo aponte para a necessidade de uma melhor compreensão dele. Segundo Paes-Sousa ${ }^{17}$, as cidades são aglomerados heterogêneos tanto socioeconomicamente quanto em relação aos perfis demográfico-epidemiológicos, de maneira que não é adequado disponibilizar modalidades de serviços de saúde tendo-se como referência apenas o tamanho da população, considerando que "populações distintas, quanto às suas necessidades de saúde, demandam serviços de saúde distintos." Verificou-se que na cidade do Rio de Janeiro, em 2010, os bairros com melhores indicadores socioeconômicos encontravam-se em nível mais avançado de envelhecimento populacional, apresentando padrão distinto de mortalidade de idosos por doenças crônicas em relação aos bairros menos avançados socioeconomicamente e menos envelhecidos, o que indica a necessidade de políticas de saúde pública específicas para bairros com cenários demográficos distintos ${ }^{18}$.

Nesse sentido, compreender os diferenciais do processo de envelhecimento populacional e suas consequências no espaço urbano pode ajudar na formulação de políticas públicas que visem o cuidado à longo prazo das doenças crônicas, bem como a distribuição e gerenciamento de recursos para a promoção da qualidade de vida, visando a redução de custos.

O presente estudo teve como objetivo descrever, através dos indicadores demográficos, o estágio do envelhecimento populacional dos bairros do município do Rio de Janeiro e verificar a existência de padrões espaciais entre eles.

\section{MÉTODOS}

Trata-se de um estudo ecológico, tendo como unidades de observação os 160 bairros que compõem o município do Rio de Janeiro, utilizando indicadores sociais e demográficos construídos a partir de informações disponíveis no Censo $2010^{19}$, utilizando as variáveis e indicadores destacados na Tabela 1.

A Porcentagem de Idosos, que indica a razão entre a quantidade de pessoas com 60 anos ou mais na População Total foi analisada em conjunto com as Porcentagens de Idosos com 60 a 69, 70 a 79 e 80 ou mais anos de idade, indicadores que permitem identificar, dentre os idosos, a porcentagem mais jovem, com idade entre 70 a 79 anos e os mais velhos. Foram analisados também três indicadores: Índice de Envelhecimento Populacional (IEP), que relaciona a população idosa com a população jovem, a Razão de Dependência de Idosos (RDI), que relaciona a população idosa com a população ativa ou não dependente, $\mathrm{e}$ a Longevidade (LI), que relaciona a população de idosos com 75 ou mais anos de idade com a população idosa. 
Tabela 1. Variáveis analisadas no estudo e método de cálculo Variável

Porcentagem de Idosos

Porcentagem de Idosos com 60 a 69 anos de idade

Porcentagem de Idosos com 70 a 79 anos de idade

Porcentagem de Idosos com 80 ou mais anos de idade

Índice de Envelhecimento Populacional - IEP

Razão de Dependência de Idosos - RDI

Longevidade - LI
Método de cálculo

$\frac{\text { População com } 60 \text { ou mais anos deidade }}{\text { PopulaçãoTotal }} \times 100$

$\frac{\text { Populaçãocom } 60 \text { a } 69 \text { anos deidade }}{\text { Populaçãocom } 60 \text { ou mais anos deidade }} \times 100$

$\frac{\text { Populaçãocom } 70 \text { a } 79 \text { anos deidade }}{\text { Populaçãocom } 60 \text { ou mais anos deidade }} \times 100$

$\frac{\text { População com } 80 \text { ou mais anos deidade }}{\text { População com } 60 \text { ou mais anos deidade }} \times 100$

$\frac{\text { População com } 60 \text { ou mais anos deidade }}{\text { População com menos de } 15 \text { anos deidade }} \times 100$

$\frac{\text { População com } 60 \text { ou mais anos deidade }}{\text { População com } 15 \text { a } 59 \text { anos deidade }} \times 100$

População com 75 ou mais anos deidade $\times 100$

População com 60 ou mais anos deidade
A análise exploratória espacial das variáveis e indicadores foi realizada através de mapas temáticos, os quais permitem observar sua distribuição no espaço, utilizando os quartis das distribuições para definir os intervalos de cores. Para detectar a existência de autocorrelação espacial, foi utilizado o Índice de Moran Global, que considera as diferenças entre os valores em cada região e a média global do atributo observado. Os resultados desse índice variam entre -1 e 1 , de maneira que valores próximos de zero indicam ausência de correlação espacial, valores próximos de 1 sugerem correlação direta e valores próximos de -1 indicam correlação inversa. Utilizou-se contiguidade de fronteira como critério de vizinhança, de maneira que bairros que dividem fronteira são considerados vizinhos, e optou-se por "unir" as ilhas ao continente para a sua definição. No caso particular da Ilha de Paquetá, foi considerada vizinhança com o centro da cidade.

A partir da detecção de autocorrelação espacial, agruparam-se os bairros com padrões distintos de envelhecimento populacional, utilizando-se o método não hierárquico K-means para dividir o conjunto de bairros, minimizando as distâncias dos indicadores internos ao grupo e maximizando as distâncias entre os grupos de bairros ${ }^{20}$. Para essa análise, foram utilizados os três indicadores: IEP, IL e RDI.

O programa computacional utilizado nas análises e construção dos mapas foi o R, na versão 3.1.2 $2^{21}$, através das bibliotecas spdep $^{22,23}$ e maptools ${ }^{24}$.

\section{RESULTADOS}

O Îndice de Envelhecimento Populacional do município do Rio de Janeiro, em 2010, era de 76,7 idosos para cada 100 jovens. Nesse ano, os idosos representavam $14,88 \%$ da população do município, sendo 7,68\% entre 60 e 69 anos de idade, 4,75\% entre 70 e 79 anos de idade e $2,45 \%$ com mais de 80 anos de idade. As mulheres apresentaram maior expectativa de vida, de maneira que, na faixa de 60 a 69 anos, 53\% eram do sexo feminino, na faixa de 70 a 79 anos essa proporção foi de $62 \%$ e na faixa etária de 80 ou mais anos, de $69,1 \%$.

A distribuição espacial da porcentagem de idosos residentes e as porcentagens dos que tinham 60 a 69,70 a 79 e 80 ou mais anos de idade são mostradas nos mapas, sendo as regiões mais escuras correspondentes aos bairros onde os valores dessas porcentagens foram mais elevados (Figura 1).

A Porcentagem de Idosos Residentes variou de 1,84\%, no bairro Gericinó, a 29,67\% em Copacabana. Observa-se um padrão de elevação nessa porcentagem no sentido oeste-leste, estando a maioria dos bairros com porcentagens mais baixas concentrados nas regiões administrativas de Realengo, Santa Cruz, Guaratiba e Barra da Tijuca, com destaque para os bairros de Campo dos Afonsos, com 1,9\%, Grumari, com 5,39\%, Itanhangá, com 6,07\% e Vargem Pequena, com 6,39\%. Os bairros caracterizados por favelas apresentaram menor porcentagem de idosos em relação aos bairros vizinhos, como é o caso de Manguinhos (6,03\%) em relação a Bonsucesso (20,02\%) e Vidigal (8,91\%) e Rocinha (5,62\%), vizinhos do Leblon (28,15\%), Gávea $(22,04 \%)$ e São Conrado (21,39\%), todos componentes do quarto quartil da distribuição dessa variável.

Os valores mais elevados concentraram-se nos bairros que compõem a região administrativa de Copacabana, Botafogo e Lagoa, situados na região litorânea que compõe a "Zona Sul", com destaque para Flamengo (29,19\%), Ipanema (28,28\%), Leblon $(28,15 \%)$ e Leme $(25,29 \%)$. Os bairros do Méier e da Tijuca se destacaram também, com porcentagem de idosos elevada: $25,58 \%$ e $24,13 \%$, respectivamente. 


\section{A. PORCENTAGEM DE IDOSOS NA POPULAÇÃO}

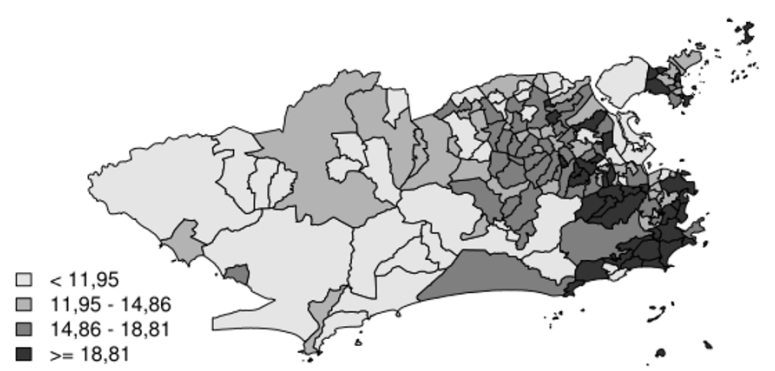

C. PORCENTAGEM DOS IDOSOS COM 70 A 79 ANOS DE IDADE

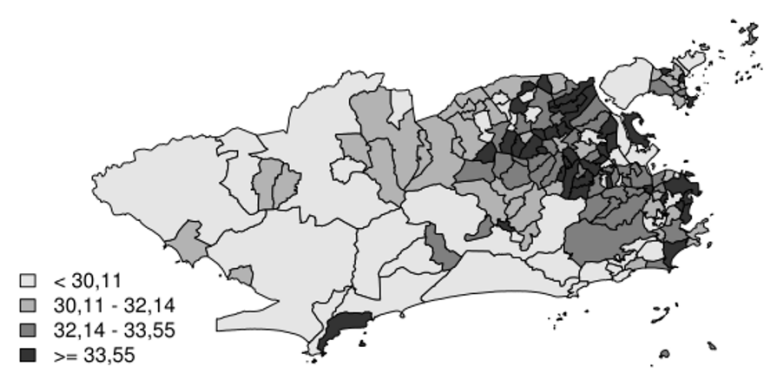

\section{B. PORCENTAGEM DOS IDOSOS COM 60 A 69 ANOS DE IDADE}

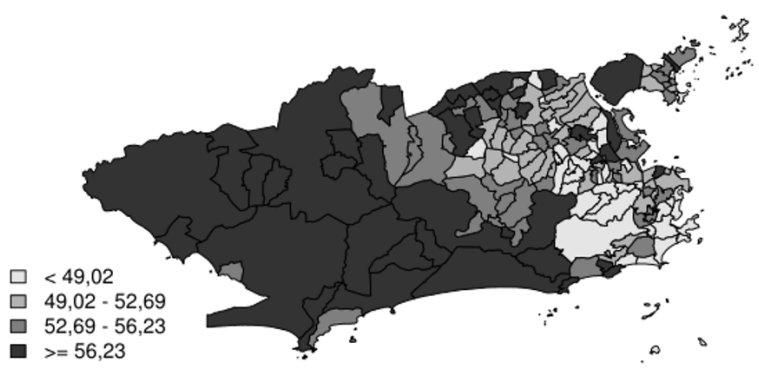

D. PORCENTAGEM DOS IDOSOS COM 80 OU MAIS ANOS DE IDADE

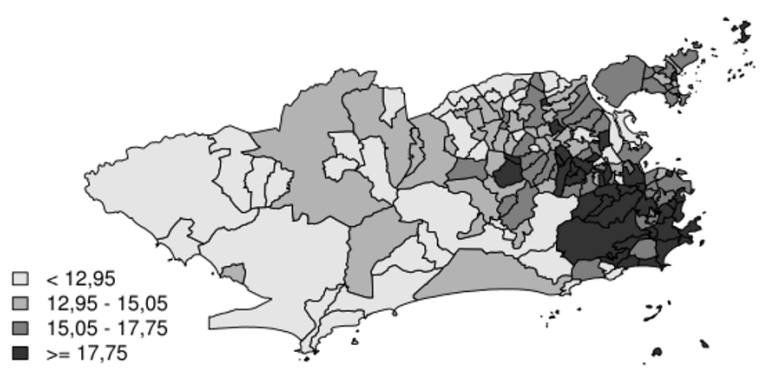

Figura 1. Distribuição espacial das variáveis: (A) Porcentagem de Idosos na população; (B) Porcentagem dos Idosos com 60 a 69 anos de idade; (C) Porcentagem dos Idosos com 70 a 79 anos de idade; e (D) Porcentagem de Idosos com 80 ou mais anos de idade

A correlação entre a Porcentagem de Idosos residentes e a Porcentagem de Idosos com 80 ou mais anos de idade foi positiva e forte $(\rho=0,83$; p-valor $<0,001)$, indicando que nos bairros onde a porcentagem de idosos residentes foi maior encontrava-se maior concentração de idosos com 80 ou mais anos de idade. Em contrapartida, observou-se uma correlação negativa entre a Porcentagem de Idosos residentes e a Porcentagem de Idosos com 60 a 69 anos ( $\rho=-0,69$; $p$-valor $<0,001)$, evidenciando que nos bairros com maior porcentagem de idosos se concentrava a menor porcentagem desses indivíduos com 60 a 69 anos de idade. Essas correlações refletiram o padrão espacial nos mapas A e D da Figura 1, com áreas mais escuras no sentido oeste-leste, e no mapa B, com padrão inverso.

Esses padrões indicaram a possibilidade de identificarmos três perfis de bairros no Rio de Janeiro em relação à porcentagem de residentes idosos: (i) os bairros que apresentam elevada porcentagem de idosos na população e nos quais se encontra a maior porcentagem de idosos com 80 ou mais anos de idade; (ii) bairros com a porcentagem de idosos na população próxima à mediana e com um maior contingente de idosos na faixa etária de 70 a 79 anos de idade; e (iii) bairros com menor porcentagem de idosos na população em geral, sendo a maioria deles "mais novos", na faixa etária entre 60 e 69 anos de idade. No entanto, como a teoria da transição demográfica descreve as transformações na estrutura etária das populações em estágios que levam em consideração o tamanho das populações jovem e adulta, além da natalidade e mortalidade, utilizaram-se os indicadores IEP, RDI e LI para caracterizar os bairros e projetar políticas públicas que atendam à população idosa atual e contribuam para que o envelhecimento da população adulta residente aconteça com melhor qualidade de vida.

O Índice I de Moran Global dos três indicadores IEP, RDI e LI foi positivo e significante (IEP: I $=0,422 \mathrm{e} \mathrm{p}$-valor $<0,001$; RDI: I = 0,350 e p-valor < 0,001; LI: 0,416 e p-valor $<0,001$ ), apontando para a existência de autocorrelação espacial global. Isso indica que bairros vizinhos apresentavam estágios semelhantes no processo de envelhecimento populacional. A distribuição dos três indicadores nos bairros do Rio de Janeiro mostra um padrão espacial similar ao observado quando se analisou apenas as porcentagens de idosos na população, com aumento no sentido oeste-leste, com concentração dos maiores valores nos bairros da "Zona Sul" (Figuras 2A, 2B e 2C)

Nesse contexto, optou-se por utilizá-los para dividir o município através do método K-means em três grupos de bairros classificados aqui como grupos I, II e III, em ordem crescente do estágio do envelhecimento populacional. O resumo dos indicadores em cada grupo está detalhado na Tabela 2 e o mapa D da Figura 2 apresenta os bairros pertencentes a cada um desses grupos.

Nessa divisão verifica-se que $60 \%$ dos bairros do Rio de Janeiro ainda se encontram em um estágio inicial do envelhecimento, alocados, portanto, no Grupo I. Nesse primeiro grupo de 97 bairros residia, segundo o censo de 2010, 66,8\% da população total e $52,9 \%$ da população idosa do município. Os índices IEP e RDI médios eram de 57,96 e 18,13, respectivamente, correspondendo a aproximadamente 2 jovens e mais de 5 indivíduos 


\section{A. ÍNDICE DE ENVELHECIMENTO POPULACIONAL}

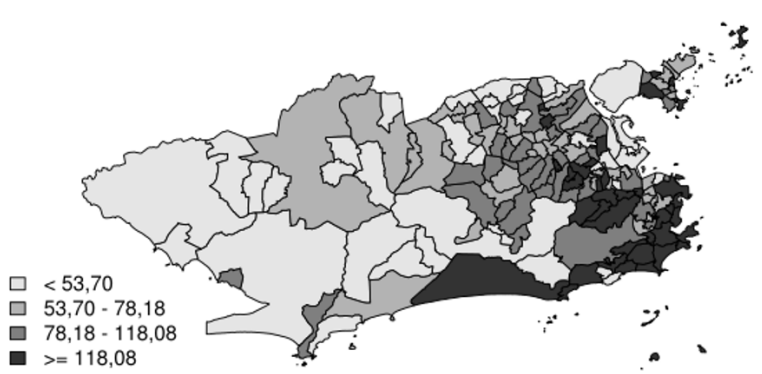

C. LONGEVIDADE DE IDOSOS

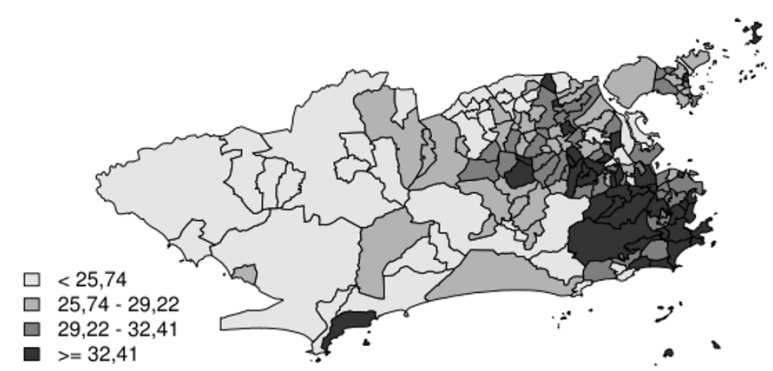

\section{B. RAZÃO DE DEPENDÊNCIA DE IDOSOS}

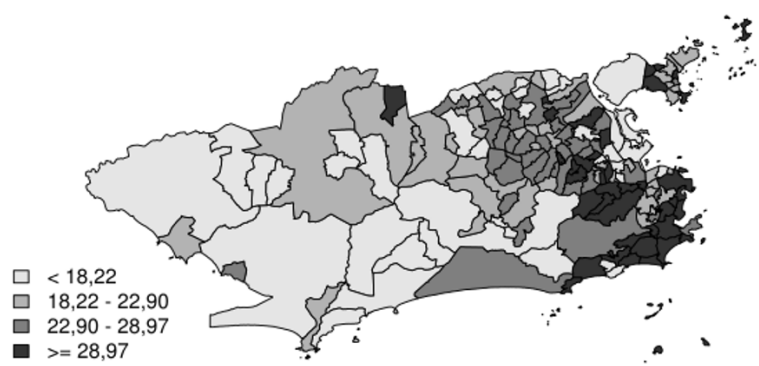

D. GRUPOS DEFINIDOS PELO MÉTODO K-MEANS

Figura 2. Distribuição espacial dos indicadores: (A) Índice de Envelhecimento Populacional; (B) Razão de Dependência de Idosos; (C) Longevidade; e (D) Grupos definidos pelo método K-means

Tabela 2. Descrição dos indicadores demográficos por grupos definidos pelo método K-means

\begin{tabular}{cccccccc} 
Grupo & \multicolumn{2}{c}{$\begin{array}{c}\text { Indice de Envelhecimento } \\
\text { Populacional }\end{array}$} & $\begin{array}{c}\text { REP } \\
\text { Média }\end{array}$ & DP & \multicolumn{2}{c}{$\begin{array}{c}\text { Razão de Dependência de } \\
\text { Idosos - RDI }\end{array}$} & \multicolumn{2}{c}{$\begin{array}{c}\text { Longevidade - LI } \\
\text { Média }\end{array}$} & DP & Média & DP & bairros \\
& 57,96 & 19,73 & 18,13 & 5,04 & 26,71 & 3,93 & $97(60,6)$ \\
I & 119,90 & 21,26 & 28,88 & 3,25 & 32,21 & 2,83 & $44(27,5)$ \\
II & 216,30 & 50,52 & 37,46 & 5,81 & 35,82 & 5,32 & $19(11,9)$ \\
III & & & & &
\end{tabular}

economicamente ativos para cada idoso. Além disso, a LI média indicava que apenas um quarto dos idosos residentes tinham 75 anos ou mais de idade. Ressalta-se que esse valor médio da longevidade foi influenciado pelo bairro de Grumari que, apesar de alocado nesse grupo apresentou o maior índice de longevidade entre os bairros do Rio de Janeiro $(44,4)$, efeito de "flutuação de pequenos números", dado que nesse bairro residiam 9 idosos, dos quais 4 tinham 75 ou mais anos de idade. O perfil do Grupo I aponta para bairros em estágio inicial do processo de envelhecimento, principalmente pela alta concentração de indivíduos nas faixas etárias jovem e adulta, comparados com a população idosa.

Entre os bairros alocados no Grupo II, os idosos correspondiam a $18,51 \%$ da população residente, sendo que mais da metade desses idosos se encontrava com 70 ou mais anos de idade. A média do IEP nesse grupo foi de 119,9, indicando que nesses bairros residia mais de um idoso para cada jovem com menos de 15 anos, enquanto a média da RDI foi de 28,88, ou seja, cerca de 3,5 indivíduos em idade economicamente ativa para cada idoso. Nesse grupo, a longevidade média indicava que 32,1\% dos idosos tinham mais de 75 anos.

Nesse contexto, os bairros que compõem o Grupo II, quando comparados aos bairros do Grupo I, se encontravam em estágio mais avançado de envelhecimento populacional, sendo sua população idosa mais "envelhecida" e quase tão representativa quanto a população residente com menos de 15 anos.

Nos bairros do Grupo III, 27,15\% da população é composta por idosos, entre os quais cerca de um quarto tinha 80 ou mais anos de idade. A média do IEP nesse grupo foi de 216,30, representando mais de 2 idosos para cada residente com menos de 15 anos e a média da RDI, 37,46, ou seja, menos de 3 indivíduos em idade potencialmente produtiva para cada idoso. A longevidade dos bairros desse grupo indicou que, em média, 36\% dos idosos tinham mais de 75 anos. Esse panorama aponta que os 19 bairros alocados nesse grupo estão em estágio avançado de envelhecimento populacional, com uma população idosa em idade avançada e predominante em relação às demais faixas etárias. 


\section{DISCUSSÃO}

As análises realizadas demonstraram que no município do Rio de Janeiro existem bairros com diferentes estágios de envelhecimento populacional. Segundo estudo recente ${ }^{18}$, os bairros que estão em um estágio mais adiantado de envelhecimento populacional são os que detêm os melhores indicadores socioeconômicos e coincidem com os que apresentam as maiores taxas de mortalidade de idosos por neoplasias, enquanto os bairros com estágio mais atrasado do envelhecimento são os que, em geral, apresentam piores indicadores socioeconômicos e maior mortalidade de idosos por doenças do aparelho circulatório. Nesse contexto, a partir da divisão dos bairros em três grupos, é possível apontar a necessidade de políticas públicas específicas de cada região da cidade de acordo com as características de sua população.

Os bairros do Grupo I, onde reside quase $70 \%$ da população do município, são os menos avançados em relação ao envelhecimento populacional e a menor concentração de idosos "mais velhos" nessa população pode ser um reflexo da maior mortalidade de idosos por doenças do aparelho circulatório, mais prevalentes na faixa etária de 60 a 69 anos. Isso pode indicar, por exemplo, a necessidade de se intensificar o controle e a prevenção das morbidades associadas ao desenvolvimento desse tipo de agravo, como hipertensão e diabetes, para garantir avanços no processo de envelhecimento populacional nesses bairros.

Além disso, nesses bairros, pelo fato de os valores de IEP e RDI indicarem um maior número de jovens e adultos em relação ao número de idosos, políticas de saúde pública com foco no envelhecimento saudável e promoção da qualidade de vida da futura população idosa devem ser desenvolvidas e implementadas desde já, de forma a garantir a sustentabilidade em longo prazo. Para isso é fundamental investir em infraestrutura, como aumento da cobertura e qualidade da rede de água e esgoto, melhoria das moradias e educação, ampliação da oferta de serviços de saúde, além de medidas específicas, como criação de áreas de convivência e de lazer, calçadas largas e com acessibilidade, descentralização de atividades econômicas, transporte público de qualidade, que evite o cansaço e stress dos deslocamentos.

Em relação aos bairros do Grupo III, nos quais observaram-se níveis mais avançados de envelhecimento, os indicadores socioeconômicos indicam menores problemas relacionados à infraestrutura urbana e o foco deverá ser na prevenção e monitoramento de doenças crônicas e na promoção da saúde como um todo, além de investimentos na formação de profissionais aptos a cuidar e atuar junto a uma população em idade cada vez mais avançada.

Os bairros do Grupo II, que se encontram em um nível intermediário de envelhecimento populacional, demandam políticas públicas que abranjam as abordagens dos grupos I e II, respeitando a heterogeneidade desse processo em cada bairro. $\mathrm{Na}$ Urca, por exemplo, em que se observou uma população adulta representativa em relação à idosa (RDI próxima à mediana) e predominância de idosos mais envelhecidos (LI elevada), as políticas públicas devem tanto contemplar os adultos mais velhos, com controle e redução de agravos e promoção da qualidade de vida, como também os idosos mais velhos, como proposto para os bairros do Grupo III. Já na Barra da Tijuca, no qual os valores da LI e da RDI foram medianos, o foco principal deve ser o idoso mais jovem e a população adulta, como proposto para os bairros do Grupo I.

A previsão das necessidades de cuidado ou atendimento deve levar em conta o padrão diferenciado de adoecimento da população atual e futura de cada local e não apenas o tamanho da população e, para isso, o Brasil têm investido na melhoria dos seus sistemas de informação e na área de vigilância epidemiológica. A disseminação de informações e resultados de pesquisas para as autoridades de saúde e para o público em geral necessita fazer parte das estratégias nacionais para identificar e enfrentar as desigualdades sociais e da saúde ${ }^{25}$.

Nesse sentido, o presente estudo identificou grupos de bairros em estágios distintos de envelhecimento populacional no município do Rio de Janeiro que demandam políticas de saúde públicas específicas para atender às necessidades atuais e futuras de sua população residente. A análise espacial dos indicadores demográficos permitiu também identificar a heterogeneidade existente no processo de transição demográfica, mesmo na segunda capital mais envelhecida do Brasil em 2010 ${ }^{19}$, apontando caminhos que podem alertar e orientar os gestores públicos na direção de um planejamento que permita que as demais cidades do país se preparem para essa nova realidade.

\section{REFERÊNCIAS}

1. Siqueira RL, Botelho MIV, Coelho FMG. A velhice: algumas considerações teóricas e conceituais. Cien Saude Colet. 2002;7(4):899-906. http://dx.doi. org/10.1590/S1413-81232002000400021.

2. Silva VL, Leal MCC, Marino JG, Marques APO. Associação entre carência social e causas de morte entre idosos residentes no Município de Recife, Pernambuco, Brasil. Cad Saude Publica. 2008;24(5):1013-23. http://dx.doi. org/10.1590/S0102-311X2008000500008. PMid:18461230.

3. Lee N, van de Graaf P, Hopkins E, O'Flaherty M. Health of the UK population in 2040. Lancet. 2015;386(9994):643-4. http://dx.doi.org/10.1016/S01406736(15)61491-X. PMid:26334147.

4. Veras RP. Prevenção de doenças em idosos: os equívocos dos atuais modelos. Cad Saude Publica. 2012;28(10):1834-40. http://dx.doi.org/10.1590/S0102311X2012001000003. PMid:23090164.

5. World Health Organization. Commission on Social Determinants of Health. Closing the gap in a generation: health equity through action on the social determinants of health. Geneva: WHO; 2008. 246 p. Commission on Social Determinants of Health Final Report.

6. Fiedler MM, Peres KG. Capacidade funcional e fatores associados em idosos do Sul do Brasil: um estudo de base populacional. Cad Saude Publica. 2008;24(2):409-15. http://dx.doi.org/10.1590/S0102-311X2008000200020. PMid:18278288. 
7. Carvalho JAM, Rodríguez-Wong LL. A transição da estrutura etária da população brasileira na primeira metade do século XXI. Cad Saude Publica. 2008;24(3):597-605. http://dx.doi.org/10.1590/S0102-311X2008000300013. PMid:18327447.

8. Braga LS, Macinko J, Proietti FA, César CC, Lima-Costa MF. Intra-urban differences in vulnerability among the elderly population. Cad Saude Publica. 2010;26(12):2307-15. http://dx.doi.org/10.1590/S0102-311X2010001200009. PMid:21243225.

9. Vieira MA, Lima RAG. Crianças e adolescentes com doença crônica: convivendo. Rev Latino-am Enfermagem. 2002;10(4):552-60. http://dx.doi. org/10.1590/S0104-11692002000400013.

10. Mariath AB, Grillo LP, Silva RO, Schmitz P, Campos IC, Medina JRP, et al. Obesidade e fatores de risco para o desenvolvimento de doenças crônicas não transmissíveis entre usuários de unidade de alimentação e nutrição. Cad Saude Publica. 2007;23(4):897-905. http://dx.doi.org/10.1590/S0102311X2007000400017. PMid:17435887.

11. Alves DB, Barbosa MTS. Desigualdades na mortalidade por doenças crônicas entre idosos e sua associação com indicadores socioeconômicos no Brasil. Rev. Bras. Ciên. Envelh. Hum. 2010;7(1):22-33. http://dx.doi. org/10.5335/rbceh.2010.003.

12. Silva MB, Barbosa MTS. Indicadores de mortalidade dos idosos nos municípios do Rio de Janeiro [Internet]. Rev. Bras. Ciên. Envelh. Hum. 2012;7(2):181-188. [citado em 2015 set 16]. Disponível em: http://www. upf.br/seer/index.php/rbceh/article/view/267

13. Miyata H, Ezoe S, Hori M, Inoue M, Oguro K, Okamoto T, et al. Japan's vision for health care in 2035. Lancet. 2015;385(9987):2549-50. http:// dx.doi.org/10.1016/S0140-6736(15)61135-7. PMid:26122147.

14. Baumgarten U. Reforming public and global health in Germany. Lancet. 2015;385(9987):2548. http://dx.doi.org/10.1016/S0140-6736(15)61145-X. PMid:26122145.

15. Araújo JD. Polarização epidemiológica no Brasil. Epidemiol. Serv. Saúde. 2012;21(4):533-8. http://dx.doi.org/10.5123/S1679-49742012000400002.

16. Frenk J, Frejka T, Bobadilla JL, Stern C, Lozano R, Sepúlveda J, et al. La transición epidemiológica en América Latina. Bol Oficina Sanit Panam. 1991;111(6):485-96. PMid:1838685.
17. Paes-Sousa R. Diferenciais intra-urbanos de mortalidade em Belo Horizonte, Minas Gerais, Brasil, 1994: revisitando o debate sobre transições demográfica e epidemiológica. Cad Saude Publica. 2002;18(5):1411-21. http://dx.doi. org/10.1590/S0102-311X2002000500034. PMid:12244374.

18. Alves D SB, Caffarena ER, Barbosa MTS. Análise espacial da mortalidade de idosos por doenças crônicas no município do Rio de Janeiro [Internet]. Rio de Janeiro: ENSP/Fiocruz; 2013 [citado em 2015 ago 20]. Disponível em: http://bvsms.saude.gov.br/bvs/publicacoes/premio2013/mestrado/ Davi\%20da\%20Silveira\%20Barroso\%20Alves.pdf

19. Instituto Brasileiro de Geografia e Estatística. Censo 2010 [Internet]. Brasília: IBGE; 2010 [citado em 2015 set 24]. Disponível em: http://censo2010.ibge. gov.br/

20. Carvalho MS, Cruz OG, Nobre FF. Perfil de risco: método multivariado de classificação sócio-econômica de microáreas urbanas-os setores censitários da região metropolitana do Rio de Janeiro. Cad Saude Publica. 1997;13(4):635-45. http://dx.doi.org/10.1590/S0102-311X1997000400007. PMid:10886905.

21. R Core Team. R: a language and environment for statistical computing [Internet]. Vienna: R Foundation for Statistical Computing; 2013 [citado em 2015 set 24]. Disponível em: http://www.R-project.org/

22. Bivand R, Piras G. Comparing implementations of estimation methods for spatial econometrics. J Stat Softw. 2015;63(18). http://dx.doi.org/10.18637/ jss.v063.i18.

23. Bivand R, Hauke J, Kossowski T. Computing the Jacobian in Gaussian spatial autoregressive models: an illustrated comparison of available methods. Geogr Anal. 2013;45(2):150-79. http://dx.doi.org/10.1111/gean.12008.

24. Bivand R, Lewin-Koh N. maptools: tools for reading and handling spatial objects. R Package Version 0.8-36. [Internet]. Vienna: R Foundation for Statistical Computing; 2015 [citado em 2015 set 24]. Disponível em: http:// CRAN.R-project.org $/$ package $=$ maptools

25. Macinko J. Advances in Brazilian public health surveillance. Cien Saude Colet. 2015;20(3):628-9. http://dx.doi.org/10.1590/1413-81232015203.20092014. PMid:25760104.
Recebido em: Dez. 09, 2015 Aprovado em: Jan. 18, 2016 